\title{
Gastrectomy as a secondary surgery for stage IV gastric cancer patients who underwent S-1-based chemotherapy: a multi-institute retrospective study
}

\author{
Tatsuo Kanda $\cdot$ Kazuhito Yajima $\cdot$ Shin-ichi Kosugi • \\ Takashi Ishikawa $\cdot$ Yoichi Ajioka $\cdot$ Katsuyoshi Hatakeyama
}

Received: 19 May 2011 / Accepted: 11 September 2011/Published online: 28 October 2011

(C) The International Gastric Cancer Association and The Japanese Gastric Cancer Association 2011

\begin{abstract}
Background Current advances in chemotherapy provide opportunities for stage IV gastric cancer patients with distant metastasis to undergo potentially curable resection. There are, however, few data on gastrectomy as a secondary surgery aimed at rendering such patients cancerfree.

Methods We investigated stage IV gastric cancer patients who underwent surgery with curative intent after S-1-based chemotherapy between 2000 and 2008. Twenty-eight patients from 12 hospitals were enrolled in this study. Factors indicating that the tumors were incurable included clinical stage T4 in 9 patients, para-aortic node metastasis in 15 , peritoneal metastasis in 7, and liver metastasis in 4 . Results Of the 28 laparotomy patients, 26 underwent complete resection with no residual tumor, obtaining a complete resection rate of $92.9 \%$. There were no in-hospital deaths or reoperations. In four patients, the primary tumor showed pathological complete response. The 1-, 3-, and 5-year overall survival rates after secondary gastrectomy were $82.1,45.9$, and $34.4 \%$, respectively, with a
\end{abstract}

T. Kanda and K. Yajima contributed equally as co-first authors.

T. Kanda $(\bowtie) \cdot$ K. Yajima · S. Kosugi · T. Ishikawa .

K. Hatakeyama

Division of Digestive and General Surgery, Niigata University

Graduate School of Medical and Dental Sciences,

Asahimachi-dori 1-757, Niigata 951-8510, Japan

e-mail: kandat@med.niigata-u.ac.jp

Y. Ajioka

Division of Molecular and Diagnostic Pathology,

Niigata University Graduate School

of Medical and Dental Sciences, Niigata, Japan median survival time of 29 months. Univariate analysis revealed histological tumor length, clinical depth of tumor invasion, number of metastatic nodes, pathological depth of tumor invasion, and pathological response to be the factors influencing patient survival after secondary surgery. On multivariate analysis, histological tumor length $(5.0 \mathrm{~cm}$ or larger) was the only significant prognostic factor (relative risk $3.23, P=0.028$ ).

Conclusions Secondary gastrectomy following S-1-based chemotherapy was a safe and effective treatment for stage IV gastric cancer. Primary tumor size is an indicator for the appropriate selection of patients for this treatment.

Keywords Chemotherapy - Gastric cancer - Prognosis . Secondary surgery $\cdot \mathrm{S}-1$

\section{Introduction}

Gastric cancer is the second most common cause of cancer death worldwide [1]. Recent advances in diagnosis and surgical techniques have improved the prognosis of early gastric cancer patients, whereas stage IV gastric cancer remains an incurable disease. Chemotherapy is the chief treatment for such unresectable tumors and/or those associated with distant metastasis. New anticancer drugs, such as S-1, paclitaxel, docetaxel, irinotecan, and oxaliplatin, have been developed in the last 20 years. Combination treatments using these potent new drugs have been actively introduced in gastric cancer chemotherapy and are contributing to significant improvements in anti-tumor responses and patient survival. In western countries, CF treatment (cisplatin and 5-FU) has played a central role in unresectable and metastatic gastric cancer therapy. DCF treatment 
(docetaxel, cisplatin, and 5-FU) was recently developed, and a clinical trial demonstrated a higher response rate, i.e., 37\%, and longer survival than $\mathrm{CF}$ treatment [2]. More recently, the REAL-2 study, a phase III clinical trial evaluating EOX treatment (epirubicin and oxaliplatin plus capecitabine), yielded a response rate of $47.9 \%$ and a median survival time (MST) of 11.2 months [3]. These results indicate that this combination could become the standard therapy for unresectable and metastatic gastric cancers.

In Japan, S-1 has played a major role in the treatment of unresectable and metastatic gastric cancers since 1999. S-1, an oral anticancer agent, consists of tegafur, gimeracil, and oteracil potassium at a molar ratio of 1:0.4:1 [4]. A phase II clinical trial on S-1 for unresectable and recurrent gastric cancers yielded a response rate of $49 \%$ and MST of 250 days [5], and when S-1 was combined with cisplatin, the response rate was $74 \%$ and MST was 383 days [6]. A phase III clinical trial conducted by the Japan Clinical Oncology Group demonstrated S-1 to be equivalent to continuous 5-FU treatment, which was the previous standard in Japan [7]. Furthermore, another large-scale phase III clinical trial showed that the overall survival of patients treated with S-1 plus cisplatin was higher than that of patients given S-1 alone [8]. Therefore, S-1-based chemotherapy is now widely accepted as the new standard treatment for unresectable and metastatic gastric cancers in Japan.

These advances in gastric cancer chemotherapy have raised new clinical issues in the treatment of incurable gastric cancer patients. During primary chemotherapy, we are presented with an opportunity to manage gastric cancer patients in whom factors indicating incurable disease have apparently disappeared or are well controlled by chemotherapy. For such patients, surgery to excise macroscopically remaining disease with curative intent may be an option. This type of surgery for gastric cancer, so-called salvage gastrectomy or secondary gastrectomy, appears to have potential benefits in terms of patient survival, but it remains unclear whether or not such a secondary surgery can be conducted safely and with certainty, and to what extent patient survival is prolonged. Additionally, as only a few studies concerning secondary gastrectomy have been reported by specialized institutions [9], it is also uncertain whether or not the results can be generalized to city hospitals.

To address these clinical issues, we conducted an investigational study on secondary gastrectomy. In this study, clinical data on secondary gastrectomy following S-1-based chemotherapy were retrospectively but systemically gathered by our research group and analyzed to reveal the surgical results, including resectability, operative morbidity, and patient survival.

\section{Patients and methods}

Study design and patients

This study was designed as a retrospective, multi-institute, investigational study. Questionnaires were sent to 36 institutions belonging to the Niigata University Trial Group of Gastric Cancer to ascertain whether or not they had performed any secondary surgeries following primary chemotherapy for patients with stage IV gastric cancer. Twenty-six institutions replied, of which 11 had cases receiving secondary gastrectomy and agreed to participate in this retrospective investigational study.

The criteria for the patient enrollment in this study were as follows: (1) newly diagnosed as having gastric adenocarcinoma, (2) clinically diagnosed with unresectable and/ or metastatic tumors, (3) underwent S-1-based chemotherapy as primary treatment, and (4) underwent laparotomy with curative intent between April 2000 and March 2008. Eleven patients who underwent S-1-based chemotherapy following palliative surgery were also included. Patients who had a history of prior chemotherapy, who had undergone chemotherapy in the neoadjuvant setting, and who were operated on for recurrent diseases and underwent surgery with palliative intent were excluded from this study.

Thirty-one patients from the aforementioned 11 hospitals and Niigata University Medical and Dental Hospital were enrolled in the present study. Two patients who underwent surgery at their request despite insufficient tumor control, and 1 patient given combined 5-FU and cisplatin before S-1-based chemotherapy were excluded. Finally, 28 patients were eligible for analysis in this study.

All patients underwent endoscopic examination, an upper gastrointestinal series, and computed tomography (CT) for clinical staging before the start of chemotherapy and when the decision regarding operative indication for secondary surgery was made. The diagnosis of adenocarcinoma was histologically confirmed based on endoscopic biopsy in all patients. The patients enrolled in this study had one or more of the following factors indicating an incurable cancer: liver metastasis, peritoneal metastasis including massive ascites, tumor invasion of adjacent organs, and para-aortic lymph node metastasis that was clearly visible on CT scans. Three surgeons (T.K., K.Y., and T.I.) conducted a retrospective central review to confirm whether or not the disease was initially incurable in the cases that the diagnosis was based on only CT findings. In this series, preoperative endoscopic ultrasonography for the evaluation of depth of tumor invasion and positron emission tomography for metastasis evaluation were performed in one patient each and staging laparoscopy was performed in two patients. 
This study was approved by the institutional review board of Niigata University Graduate School of Medical and Dental Sciences (no. 853-09).

\section{Primary chemotherapy}

After the tumors had been diagnosed as unresectable or incurable, patients received S-1 as primary chemotherapy. Treatment regimens varied and modified among the institutions. Four S-1-based treatment regimens were used in this study: S-1 alone, S-1 plus cisplatin, S-1 plus irinotecan, and S-1 plus paclitaxel. The S-1 alone regimen was S-1 (TS- ${ }^{\circledR}$, Taiho Pharmaceutical Co., Ltd., Tokyo, Japan) administered daily in a 4-week-on and 2-week-off schedule. The S-1 plus cisplatin regimen was $\mathrm{S}-1$ administered daily for 3 weeks, followed by a 2-week rest, with cisplatin at $60 \mathrm{mg} / \mathrm{m}^{2}$ given on day 8 [6]. S-1 plus paclitaxel $\left(\right.$ Taxol $^{\circledR}$, Bristol-Myers K.K., Tokyo, Japan) was S-1 administered for 14 consecutive days, followed by a 1 -week rest, with paclitaxel at $50 \mathrm{mg} / \mathrm{m}^{2}$ on day 1 and day 8 [10]. The S-1 plus irinotecan (Topotecin ${ }^{\circledR}$, Daiichi Pharmaceutical Co. Ltd., Tokyo, Japan) regimen was oral S-1 administered at a dose of $80 \mathrm{mg} / \mathrm{m}^{2}$ per day for 3 weeks, followed by a 2-week rest, with irinotecan at $80 \mathrm{mg} / \mathrm{m}^{2}$ given on day 1 and day 15 [11]. For all S-1 regimens, S-1 was administered orally based on the patient's body surface area (BSA; BSA $<1.25 \mathrm{~m}^{2}, 80 \mathrm{mg}$ / day; $1.25 \leq \mathrm{BSA}<1.5 \mathrm{~m}^{2}, 100 \mathrm{mg} /$ day, $1.5 \mathrm{~m}^{2} \leq \mathrm{BSA}$, $120 \mathrm{mg} /$ day) [5].

The toxicity of chemotherapy was assessed according to the National Cancer Institute-Common Toxicity Criteria for Adverse Events (NCI-CTCAE version 3.0). The clinical response of measurable metastatic tumors was evaluated based on the guidelines of the Response Evaluation Criteria in Solid Tumors (RECIST) [12]. Complete regression of immeasurable disease, such as malignant ascites, was confirmed by disappearance of ascites on CT scans. Complete response (CR), partial response (PR), no change (NC), and progressive disease (PD) were defined according to the response assessment criteria proposed in the Japanese Classification of Gastric Cancer, 2nd English edition, (JCGC) [13] and RECIST.

Secondary surgery and the surgical outcomes

In this retrospective study, secondary gastrectomy was defined as surgery with curative intent, i.e., surgery aiming at macroscopic complete resection of tumors that were controlled but remained after chemotherapy. To ascertain feasibility, we included surgeries that resulted in incomplete resection or exploration in this study. Patient selection and the timing of secondary surgery were determined by gastrointestinal surgeons, radiologists, and clinical oncologists or gastroenterologists at each hospital. The surgical procedure was finally selected by the surgeons assigned to perform the operation. To ascertain the safety of secondary gastrectomy, we evaluated the operative time, blood loss, operative mortality, morbidity, and hospital stay. Operative mortality was defined as in-hospital death within 30 days of the surgery, and morbidities were defined as complications that required additional treatment or an extended hospital stay. Surgical morbidities were evaluated by reviewing medical charts and graded on a scale of 1-4, according to the NCI-CTCAE version 3.0.

\section{Histopathological examination}

Resected specimens were histopathologically analyzed in each hospital to evaluate the extent of residual disease, effect of chemotherapy, and disease stage. In the event of there being no residual cancer cells in the main section, areas of the whole stomach were cross-sectioned serially to assure the histological diagnosis of CR. Clinical, surgical, and final pathological staging was determined for each patient who underwent gastrectomy. As we regarded paraaortic lymph node metastasis as a distant metastasis, the AJCC Cancer Staging Manual, sixth edition, was used for tumor descriptions and disease staging in the present study [14]. Cancers involving the upper, middle, and lower thirds of the stomach were defined as cancers involving the "whole body" of the stomach. The histological grade of tumor regression was classified based on the JCGC [15]. Grade 1a was defined as shrinkage of primary tumor, which was less than one-third of the original tumor size, grade $1 b$ as less than two-thirds shrinkage, grade 2 as more than twothirds shrinkage but incomplete, and grade 3 as complete regression.

Follow-up data, survival curves, and statistical analysis

Medical charts, surgical records, and pathological reports were reviewed, and data of all 28 eligible patients were collected in case report forms. Data on prognoses were available until death or the date of the last follow-up. Follow-up periods from initiation of chemotherapy ranged from 11 to 104 months (median 37.5 months). Survival curves were constructed using the Kaplan-Meier method, and differences in survival were evaluated with the logrank test. To determine factors that would possibly influence postoperative survival, 19 variables were entered for univariate and multivariate analyses. As imaging data on tumor length were not available for all cases, the histologically measured largest tumor length in the surgical specimens was used for the analyses. The Cox proportional hazards regression model was used for identifying factors that were independently associated with postoperative 
survival. Stepwise variable selection was used with entry and removal limits of $P<0.10$ and $P>0.15$ in the Cox model. The stability of this model was confirmed using a step-backward and step-forward fitting procedure. All statistical evaluations were performed using the SPSS 11.5J software package (SPSS Japan Inc., Tokyo, Japan). A $P$ value less than 0.05 (two-tailed) was considered statistically significant.

\section{Results}

Clinicopathological characteristics

Ages of the patients at the time of secondary gastrectomy ranged from 48 to 79 years (median 65.5 years). Fifteen patients were men and 13 were women. Clinicopathological characteristics at initiation of chemotherapy are shown in Table 1. Factors indicating incurable tumors were clinical stage T4 in 9 patients, para-aortic node metastasis in 15, peritoneal metastasis in 7 (including 3 with massive ascites and 1 with a Krukenberg tumor), and liver

Table 1 Clinicopathological characteristics of 28 patients who underwent secondary gastrectomy at initiation of chemotherapy

\begin{tabular}{lc}
\hline Characteristic & $\begin{array}{c}\text { Number } \\
\text { of patients }\end{array}$ \\
\hline Sex & 15 \\
Men & 13 \\
Women & \\
Tumor location & 6 \\
Upper third of stomach & 6 \\
Middle third of stomach & 13 \\
Lower third of stomach & 3 \\
Whole body of stomach & \\
Factors indicating incurable disease & 9 \\
Tumor invasion to adjacent organ(s) & 15 \\
Para-aortic node metastasis & 7 \\
Peritoneal metastasis & 4 \\
Liver metastasis & \\
Histological types by biopsy specimens & 11 \\
Intestinal type & 17 \\
Diffuse type & \\
Macroscopic type, JCGC & 8 \\
Type 1, 2 & 20 \\
Type 3, 4 & 5 \\
Prior surgery & \\
Gastrojejunostomy & \\
Exploratory laparotomy & \\
Tube jejunostomy & \\
\hline & \\
\hline
\end{tabular}

JCGC Japanese classification of gastric carcinoma metastasis in 4. Seven patients had two factors indicating incurable tumors. T4 tumors were estimated to have invaded the pancreas in eight patients and the lateral segment of the liver in one. Among those with liver metastases, three patients had multiple liver tumors and one had a solitary liver tumor.

Primary chemotherapy

Chemotherapeutic regimens and their effects are summarized in Table 2. An S-1 combination regimen with cisplatin was administered to 22 patients, with paclitaxel, to 4 , and with irinotecan, to 1 . One patient received S-1 alone because of low renal function. The median number of treatment courses with S-1-based chemotherapy was three (range 2-5), and the median time of primary chemotherapy was 108 days (range 42-357 days). Of the 28 patients who underwent S-1-based chemotherapy, 3 received the chemotherapy with weekly paclitaxel regimen while the disease was well controlled [16]. The weekly paclitaxel regimen was given in 2,8 , and 10 courses. Clinical responses to the primary chemotherapy were $\mathrm{CR}$ in 2 patients and PR in 26. A total of nine grade-3 treatmentrelated adverse events were seen in seven patients; neutropenia in three, anorexia in three, diarrhea in two, and rash in one. No patients had grade-4 treatment-related adverse events.

Table 2 Chemotherapy and effects in 28 eligible patients

\begin{tabular}{ll}
\hline & Number of patients \\
\hline Primary chemotherapy regimens & \\
S1 alone & 1 \\
S1 plus cisplatin & 22 \\
S1 plus paclitaxel & 4 \\
S1 plus irinotecan & 1 \\
Median no. of courses (range) & \\
S1 alone & 3 courses \\
S1 plus cisplatin & 3 courses (range 2-5 courses) \\
S1 plus paclitaxel & 4.5 courses (range 2-5 courses) \\
S1 plus irinotecan & 3 courses \\
Median time of primary & 108 days (42-357 days) \\
chemotherapy (range) & \\
Clinical response & 2 \\
CR & 26 \\
PR & \\
Adverse events (grade 3/4) & 3 \\
Neutropenia & 3 \\
Anorexia & 2 \\
Diarrhea & \\
Rash & \\
\hline
\end{tabular}

$C R$ complete response, $P R$ partial response 
Surgical outcomes

The surgical procedures and outcomes are shown in Table 3. Median time between the initiation of primary chemotherapy and secondary surgery was 130 days (range 59-391 days). All patients underwent gastrectomy: total gastrectomy in $12(42.9 \%)$ and distal gastrectomy in 16 $(57.1 \%)$ patients. A D2 lymphadenectomy was performed in 17 patients, D1 lymphadenectomy in one, D2 plus paraaortic nodal dissection in 5, and D3 lymphadenectomy in 5 . Other organs resected at the time of gastrectomy were as follows: spleen in ten patients, gallbladder in seven, liver in two, transverse colon in two, lower esophagus with median phrenotomy in two, and caudal pancreas in one. Complete resection with no residual tumor was achieved in 26 of 28 patients (complete resection rate 92.9\%), microscopic residual tumor status in one (positive for peritoneal

Table 3 Surgical procedures and outcomes of 28 patients who underwent secondary gastrectomy

\begin{tabular}{|c|c|}
\hline & Number of patients \\
\hline \multicolumn{2}{|l|}{ Surgical procedures } \\
\hline Total gastrectomy & 12 \\
\hline Distal gastrectomy & 16 \\
\hline \multicolumn{2}{|l|}{ Lymphadenectomy, JCGC } \\
\hline D1 & 1 \\
\hline D2 & 17 \\
\hline D2 plus PAND & 5 \\
\hline D3 & 5 \\
\hline \multicolumn{2}{|l|}{ Combined resections } \\
\hline Spleen & 10 \\
\hline Gallbladder & 7 \\
\hline Liver & 2 \\
\hline Transverse colon & 2 \\
\hline Lower esophagus & 2 \\
\hline Caudal pancreas & 1 \\
\hline \multicolumn{2}{|l|}{ Residual tumor status } \\
\hline R0 & 26 \\
\hline $\mathrm{R} 1$ & 1 \\
\hline $\mathrm{R} 2$ & 1 \\
\hline Median operative time (range) & $265.5 \min (124-588 \mathrm{~min})$ \\
\hline Median blood loss (range) & $418.5 \mathrm{ml}(35-2168 \mathrm{ml})$ \\
\hline Median hospital stay (range) & 18 days (9-86 days) \\
\hline Morbidity & 4 \\
\hline Pancreatic fistula & 2 \\
\hline Intra-abdominal abscess & 1 \\
\hline Anastomotic leakage & 1 \\
\hline
\end{tabular}

D1 dissection of all group 1 nodes, $D 2$ dissection of all group 1 and group 2 nodes, D3 dissection of all group 1, group 2, and group 3 nodes, JCGC Japanese classification of gastric carcinoma, PAND para-aortic nodal dissection, $R O$ no residual tumor, $R 1$ microscopic residual tumor, $R 2$ macroscopic residual tumor washing cytology) and macroscopic residual tumor in one (peritoneal metastasis).

Median operative time was $262.5 \mathrm{~min}$ (range 124-588 min) and median blood loss was $418.5 \mathrm{ml}$ (range 35-2,168 ml). There were neither in-hospital deaths nor operative mortalities. On the other hand, there were four postoperative complications of grade 2 or more severe; pancreatic fistula in two patients, intra-abdominal abscess in the left subphrenic space in one, and anastomotic leakage of an esophago-jejunostomy in one. No patients required surgical or radiological intervention for operative complications. Median hospital stay after surgery was 18 days (range 9-86 days).

\section{Postoperative chemotherapy}

In this study, 27 of 28 patients were given additional chemotherapy after secondary surgery. S-1-based regimens were selected in 22 patients; S-1 alone in 15 and S-1-based combination treatment in 7 . Weekly paclitaxel treatment was administered to four patients, and sequential methotrexate plus 5-FU to one. The median number of courses of postoperative S-1-based chemotherapy was four (range $1-14$ courses).

\section{Pathological findings}

Table 4 summarizes the pathological responses of the tumors to treatment in the 28 patients who underwent secondary gastrectomy. Pathological stage T4 tumors were seen in two patients: one was histologically proven to have invaded the lateral segment of the liver and the other the pancreatic head. Median tumor length was $5.0 \mathrm{~cm}$ (range $0-15.0 \mathrm{~cm}$ ). Pathological para-aorta lymph node metastasis was demonstrated in six patients. The median number of metastatic nodes was 3.0 (range $0-14$ ). Histologically complete regression of primary tumors (grade 3 ) was found in four patients.

Patient survival and prognostic factors

For all 28 patients, the respective 1-, 3-, and 5-year overall survival rates from initiation of chemotherapy were 96.4, 53.3, and 34.3\%, with a MST of 37 months (Fig. 1). The respective 1-, 3-, and 5-year overall survival rates after secondary gastrectomy were $82.1,45.9$, and $34.4 \%$, with a MST of 29 months (Fig. 2). Tumor recurrence and relapse were seen in 18 patients and 21 areas: liver metastasis occurred in 5 patients, peritoneal recurrence in 5 , bone metastasis in 1 , and lymph node recurrence in 10 . The ten with lymph node recurrence included four, two, three, and one patient, respectively, with positive locoregional, hepatic hilum, para-aortic, and mediastinal lymph nodes. 
Table 4 Pathological findings in 28 patients who underwent secondary gastrectomy

\begin{tabular}{ll}
\hline Variable & Number of patients \\
\hline Depth of tumor invasion, AJCC & \\
CR & 4 \\
pT1 & 3 \\
pT2 & 11 \\
pT3 & 8 \\
pT4 & 2 \\
Node metastasis, AJCC & \\
pN0 & 5 \\
pN1 & 17 \\
pN2 & 6 \\
Distant metastasis, AJCC & \\
pM1 (para-aortic lymph nodes) & 6 \\
pM1 (liver) & 2 \\
pM1 (positive for washing cytology) & 2 \\
pM1 (peritoneum) & 1 \\
Median number of metastatic nodes (range) & $3(0-14)$ \\
Grade of pathological response (primary tumor) \\
Grade 1a & 10 \\
Grade 1b & 7 \\
Grade 2 & 7 \\
Grade 3 & 4 \\
\hline ACC America Joint Commitee & \\
\hline
\end{tabular}

AJCC American Joint Committee on Cancer, $C R$ complete response, $p T$ pathological T, $p N$ pathological N, $p M$ pathological M, Grade $1 a$ less than one-third tumor shrinkage, grade $1 b$ less than two-thirds tumor shrinkage, grade 2 more than two-thirds tumor shrinkage but incomplete, grade 3 complete regression

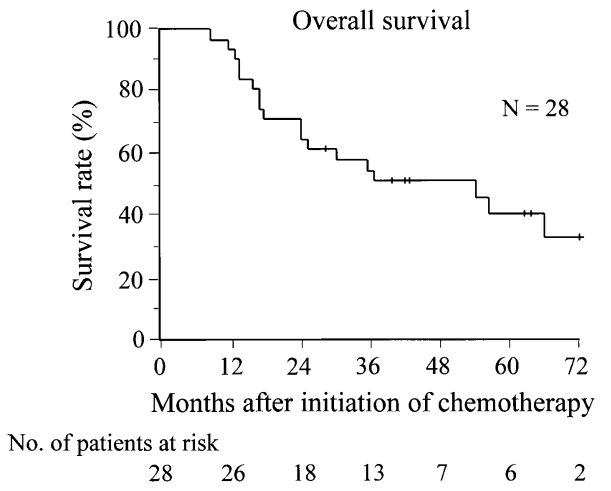

Fig. 1 Kaplan-Meier curves for overall survival from the time of initiation of chemotherapy for 28 patients who underwent secondary gastrectomy. The 1-, 3-, and 5-year overall survival rates from the initial chemotherapy were $96.4,53.3$, and $34.3 \%$, respectively, with a median survival time of 37 months

On univariate analysis, histological tumor length $(P=0.002)$, clinical depth of tumor invasion $(P=0.027)$, pathological depth of tumor invasion $(P=0.033)$, number of metastatic nodes $(P=0.005)$, and pathological response (grades 0,1 vs. grades 2,$3 ; P=0.038$ ) were factors that

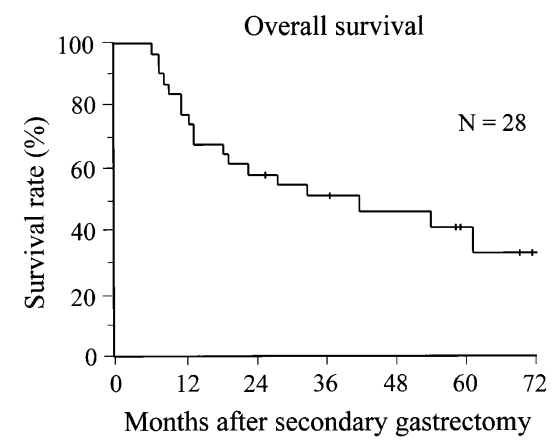

No. of patients at risk

$\begin{array}{lllllll}28 & 23 & 15 & 11 & 7 & 4 & 2\end{array}$

Fig. 2 Kaplan-Meier survival estimates for overall survival from the time of secondary gastrectomy for the 28 eligible patients. The 1-, 3-, and 5-year overall survival rates after secondary gastrectomy were $82.1,45.9$, and $34.4 \%$, respectively, with a median survival time of 29 months

significantly influenced postoperative patient survival (Table 5). In Fig. 3, we show the Kaplan-Meier survival curves of the 28 patients according to these five factors. On multivariate analysis, histological tumor length (relative risk 3.23 ; 95\% confidence interval $1.13-9.18, P=0.028$ ) was identified as an independent prognostic factor (Table 6).

\section{Discussion}

Advances in gastric cancer chemotherapy, such as the introduction of new anticancer agents, have made macroscopic complete resection possible for some of the patients presenting with otherwise unresectable or metastatic gastric cancer for whom surgical resection had not been indicated at the first clinic visit. Surgical resection of a residual tumor in such patients, or the so-called salvage gastrectomy or secondary gastrectomy, has been sporadically reported since 2001: all of those reports have described surgical success and satisfactory prognosis [1719]. However, such case reports include publication bias and must be carefully evaluated. Although data quality must be improved to accurately determine clinical validity, systematic studies on secondary gastrectomy after treatment with new anticancer drugs have been quite limited: from an intensive literature search, we found that only the case-series study by Ishigami et al. [9] addressed this issue.

Ishigami et al. reported the results of secondary gastrectomy in 18 patients. Their report indicated that complete resection (R0) was achieved with no serious postoperative complications in 14 of the 18 patients, and that the MST of the 14 patients was long, i.e., 997 days. These satisfactory results from their pioneering work seem to support the usefulness of secondary gastrectomy for 
Table 5 Univariate analysis of survival rates among 28 patients who underwent secondary gastrectomy, according to selected prognostic variables

$c T, c N, c M 1$ clinical stage

before initiation of chemotherapy, $P R$ partial response, $C R$ complete response, Distal distal gastrectomy, Total total gastrectomy

${ }^{\text {a }}$ Histologically measured in surgical specimens

\begin{tabular}{|c|c|c|c|}
\hline \multirow[t]{2}{*}{ Variable } & \multicolumn{3}{|c|}{ Univariate analysis } \\
\hline & No. of patients & 3 -year survival rate $(\%)$ & $P$ value \\
\hline Sex (men vs. women) & $15 / 13$ & $38.9 / 53.8$ & 0.588 \\
\hline Age ( $<70$ vs. $\geq 70$ years $)$ & $14 / 14$ & $42.9 / 50.0$ & 0.804 \\
\hline cT (T2 vs. T3, T4) & $7 / 21$ & $68.6 / 38.1$ & 0.027 \\
\hline cN (N0 vs. N1, N2) & $5 / 23$ & $38.5 / 51.9$ & 0.126 \\
\hline cM; lymph nodes (absence vs. presence) & $13 / 15$ & $53.8 / 40.0$ & 0.090 \\
\hline cM1; liver (absence vs. presence) & $23 / 4$ & $45.1 / 50.0$ & 0.690 \\
\hline cM1; peritoneum (absence vs. presence) & $21 / 7$ & $55.0 / 28.6$ & 0.238 \\
\hline No. of factors indicating incurable disease (1 vs. 2 ) & $21 / 7$ & $47.1 / 42.9$ & 0.387 \\
\hline Clinical response (PR vs. CR) & $26 / 2$ & $45.6 / 50.0$ & 0.519 \\
\hline Types of gastrectomy (distal vs. total) & $16 / 12$ & $49.2 / 41.6$ & 0.801 \\
\hline Pathological type (tub1, tub2 vs. por1, por2, sig) & $11 / 17$ & $54.5 / 39.7$ & 0.524 \\
\hline Macroscopic type (type 1,2 vs. type 3,4 ) & $8 / 20$ & $72.9 / 35.0$ & 0.211 \\
\hline Tumor length $^{\mathrm{a}}$ ( $\leq 5.0$ vs. $\left.>5.0 \mathrm{~cm}\right)$ & $15 / 13$ & $61.4 / 25.0$ & 0.002 \\
\hline pT (CR, T1, T2 vs. T3, T4) & $18 / 10$ & $55.0 / 30.0$ & 0.033 \\
\hline pN (N0 vs. N1, N2) & $5 / 23$ & $60.0 / 42.7$ & 0.982 \\
\hline pM1; lymph nodes (absence vs. presence) & $22 / 6$ & $44.6 / 50.0$ & 0.654 \\
\hline No. of metastatic nodes ( $<3$ vs. $\geq 3$ ) & $16 / 12$ & $70.6 / 27.3$ & 0.005 \\
\hline Pathological response (grade $0,1 \mathrm{a}, 1 \mathrm{~b}$ vs. 2,3 ) & $17 / 11$ & $35.3 / 63.4$ & 0.038 \\
\hline Pathological response (grade $0,1 \mathrm{a}$ vs. $1 \mathrm{~b}, 2,3$ ) & $10 / 18$ & $30.0 / 55.0$ & 0.067 \\
\hline Residual tumor status (R0 vs. R1, R2) & $26 / 2$ & $49.5 / 0$ & 0.059 \\
\hline
\end{tabular}

patients with highly advanced gastric cancer after the introduction of new anticancer drugs. However, theirs was a retrospective study performed at a single university hospital and included only 18 patients. Therefore, whether their results can be generalized and whether secondary gastrectomy is a viable therapeutic option for highly advanced gastric cancer remain unclarified. The present multi-institute, retrospective study was performed to obtain higher-quality data needed to address these clinical issues.

This multi-institute study revealed clinically important points related to secondary gastrectomy. First, the findings on the safety and feasibility of secondary gastrectomy were confirmed. In this study, there were neither in-hospital deaths nor reoperations in the 28 patients. Only four (14.3\%) experienced grade 2 or more severe postoperative complications. Ishigami et al. reported only one postoperative complication, anastomotic leak, among their 18 patients, and this result is consistent with ours. A multiinstitute clinical study on radical surgery for gastric cancer in Japanese patients (JCOG9501) [20] showed a complication incidence of $20.9 \%$ in the patient group with D2 lymph node dissection and $28.1 \%$ in the group that underwent an extended operation with aortic lymph node dissection. Other case-series studies on radical surgery for gastric cancer also showed postoperative complication incidence in the range of $17.9-46 \%$ [21, 22]. These findings indicate that the incidence of complications in our patient series is not significantly higher than that in patients who underwent conventional radical surgery for gastric cancer. As for resectability, the rate of complete resection was high in our study, $92.9 \%$. These satisfactory safety and feasibility results may be explained in part by the frequent use of D2 dissection in gastric cancers and the resulting high technical standard for the resection of advanced gastric cancers in Japan. Furthermore, the results may also be attributable to careful patient selection by investigators at study institutions because secondary gastrectomy is indicated for only high-risk patients who have undergone longterm chemotherapy for highly advanced gastric cancer.

Second, the present study provided data on the prognosis of patients who underwent secondary gastrectomy. Ishigami et al. reported a long MST of 26 months from the start of chemotherapy in 18 patients who underwent secondary gastrectomy and 33 months in 14 patients in whom R0 resection could be performed. Unexpectedly, in their study, as the survival period after secondary gastrectomy was not analyzed, the effect of resection on survival could not be determined. To address this issue, we analyzed not only survival after the start of chemotherapy, but also that after secondary gastrectomy.

Our results indicate that patients who underwent secondary gastrectomy have good prognoses, with MST after the start of chemotherapy of 37 months and MST after secondary gastrectomy of 29 months. However, these good 
A Histological tumor length

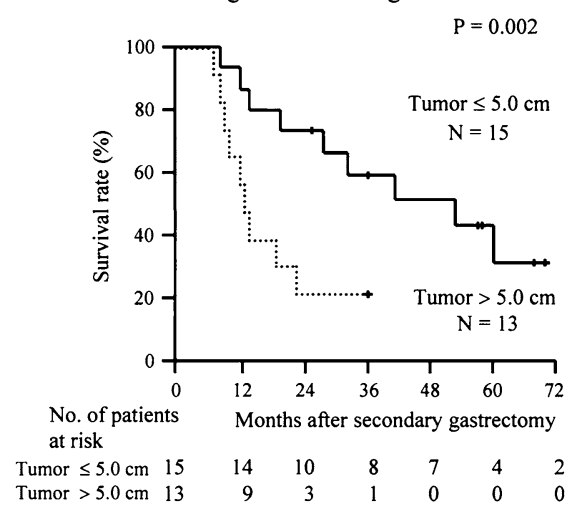

B Clinical depth of tumor invasion

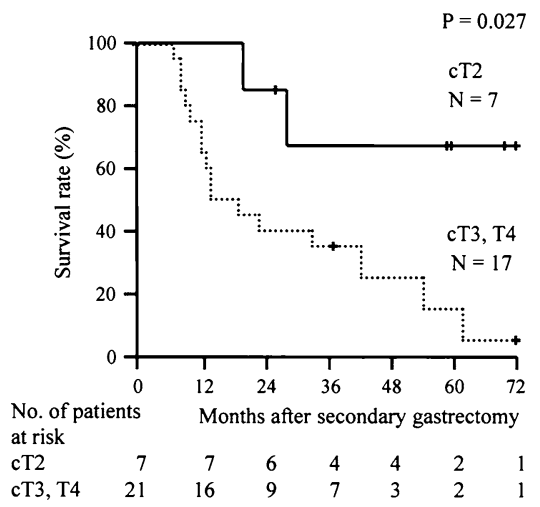

C Pathological depth of tumor invasion

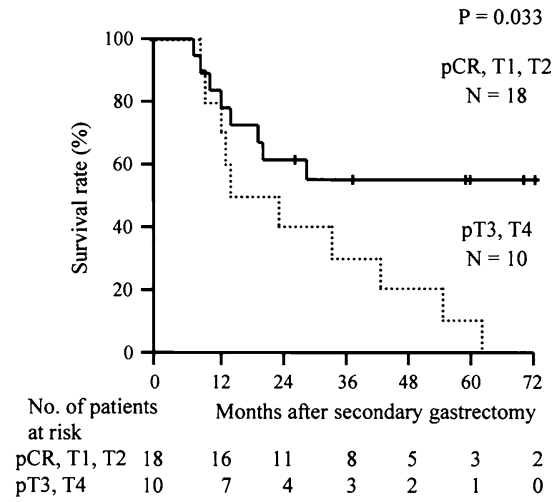

D No. of metastatic nodes

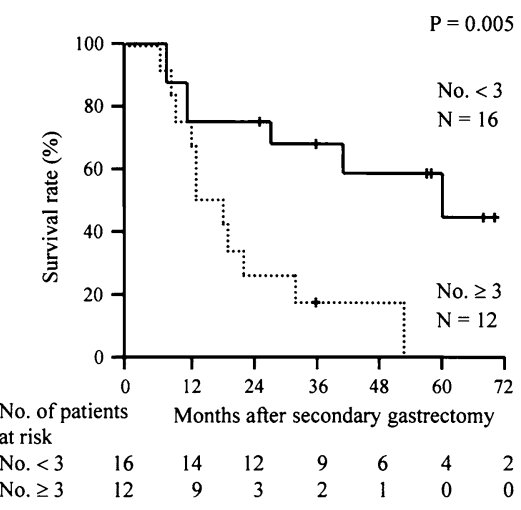

E Pathological response

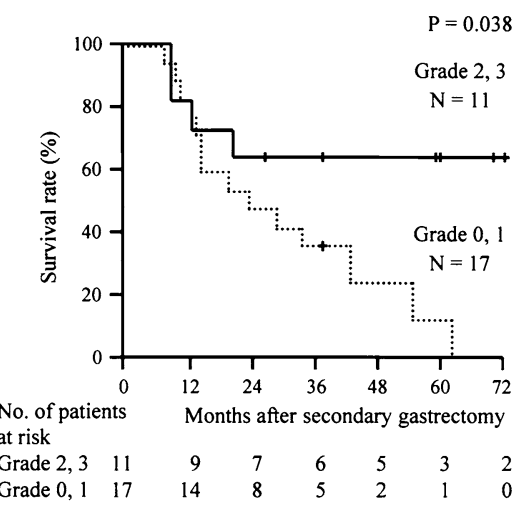

Fig. 3 Kaplan-Meier survival estimates for overall survival from the time of secondary gastrectomy for the 28 eligible patients, according to histological tumor length (a), clinical depth of tumor invasion (b), pathological depth of tumor invasion (c), number of metastatic nodes (d), and pathological response (e)

Although neither the study of Nashimoto et al. nor ours demonstrated that surgical resection per se improved the final prognosis, it should be noted that both studies showed that long-term survival could be achieved through comprehensive tumor control based on a combination of high responsiveness to chemotherapy and surgical resection of residual tumors.

Third, in the present study, we characterized patients who are likely to benefit from secondary gastrectomy. Determining which patients would benefit from secondary gastrectomy is an important issue in establishing multimodality treatment. Univariate analysis in the present study revealed that the factors affecting prognosis included histological tumor length, the clinical depth of tumor invasion, the pathological depth of tumor invasion, the number of metastatic lymph nodes, and the pathological response of the primary tumor. Multivariate analysis showed histological tumor length to be the only significant prognostic factor. These findings indicate that, no matter what factors indicating incurable disease were initially present, the status of the reduced and remaining tumor following chemotherapy can influence postoperative prognosis of the 
Table 6 Multivariate analysis of prognostic factors in 28 patients who underwent secondary gastrectomy

\begin{tabular}{|c|c|c|c|}
\hline \multirow[t]{2}{*}{ Variables } & \multicolumn{3}{|c|}{ Multivariate analysis } \\
\hline & $\mathrm{RR}$ & $95 \% \mathrm{CI}$ & $P$ value \\
\hline \multicolumn{3}{|l|}{ Tumor length $^{\mathrm{a}}$} & 0.028 \\
\hline$\leq 5.0 \mathrm{~cm}$ & 1.00 & & \\
\hline$>5.0 \mathrm{~cm}$ & 3.23 & $1.13-9.18$ & \\
\hline \multicolumn{3}{|l|}{$\begin{array}{l}\text { Clinical depth } \\
\text { of tumor invasion }\end{array}$} & 0.971 \\
\hline $\mathrm{cT} 2$ & 1.00 & & \\
\hline $\mathrm{cT} 3, \mathrm{~T} 4$ & 1.02 & $0.34-3.11$ & \\
\hline \multicolumn{3}{|l|}{$\begin{array}{l}\text { Pathological depth } \\
\text { of tumor invasion }\end{array}$} & 0.609 \\
\hline $\mathrm{pCR}, \mathrm{T} 1,2$ & 1.00 & & \\
\hline pT3, 4 & 1.84 & $0.18-19.3$ & \\
\hline \multicolumn{3}{|c|}{ No. of metastatic nodes } & 0.153 \\
\hline$<3$ & 1.00 & & \\
\hline$\geq 3$ & 2.08 & $0.76-5.65$ & \\
\hline \multicolumn{3}{|l|}{ Pathological response } & 0.330 \\
\hline Grade 2,3 & 1.00 & & \\
\hline Grade 0,1 & 1.95 & $0.51-7.43$ & \\
\hline
\end{tabular}

patients. Because the present study was a multi-institute and retrospective one, diagnostic imaging data on tumor length were not available. Instead, data on the histological size of tumors from pathological specimens were used for analysis. Therefore, the criterion of tumor length of $5 \mathrm{~cm}$ or less, which was derived from the data, cannot be directly used in selecting patients suitable for secondary gastrectomy, although it may serve as one of the important indicators for this surgery.

In the present study, we carefully defined secondary surgery and included all patients who underwent this procedure in the participating institutions regardless of their results. Nevertheless, selection bias could not be excluded. Moreover, although it is clinically important to determine the incidence of patients who underwent such a secondary surgery, the total number of patients with incurable gastric cancer who underwent S-1-based chemotherapy at the 12 participating institutions was not determined because this study was a retrospective one: it is presumable that the number of patients who are candidates for secondary gastrectomy is very small, considering that only 28 patients were found in 12 hospitals over the 8-year period despite the high incidence of gastric cancer in Japan. To precisely know the incidence and improve data quality, a prospective clinical study allowing intent-to-treat analysis is essentially required.
Finally, the present multi-institute, retrospective study provided useful information on clinically important issues regarding secondary gastrectomy. Secondary gastrectomy could be performed safely in carefully selected patients and was associated with a sufficiently high complete resection rate. The prognoses of patients who underwent secondary gastrectomy were good: this procedure could be indicated particularly for patients with a primary tumor length of $5.0 \mathrm{~cm}$ or less. These promising results justify the need for a randomized prospective study to clarify the clinical significance of secondary gastrectomy in patients with highly advanced gastric cancer who responded to chemotherapy.

Acknowledgments We thank Drs. M. Hasegawa (Niigata Prefectural Central Hospital), H. Okamoto (Niigata Prefecture Yoshida Hospital), S. Tanaka (Niigata Prefecture Koide Hospital), Y. Sakai, N. Musha (Saiseikai Niigata Daini Hospital), E. Sunami (Shirone Kense Hospital), T. Suda (Nippon Dental University School of Life Dentistry at Niigata, Medical Hospital), T. Kobayashi (Niigata Rinko Hospital), N. Shimakage, T. Kakuta (Japanese Red Cross Nagaoka Hospital), M. Ohashi (Tokyo Metropolitan Cancer and Infectious Diseases Center Komagome Hospital), K. Endo, T. Naito (Akita Kumiai General Hospital), and K. Ueki (Kariwagun General Hospital) for assistance with the data collection. We wish to thank Dr. K. Akazawa, Professor of the Division of Information Science and Biostatistics, Niigata University Graduate School of Medical and Dental Sciences, for helpful advice on statistical analysis.

\section{References}

1. Parkin DM. Global cancer statistics. 2000. CA cancer J Clin. 2005;55:74-108.

2. Van Cutsem E, Moiseyenko VM, Tjulandin S, Majlis A, Constenla M, Boni C, et al. Phase III study of docetaxel and cisplatin plus fluorouracil compared with cisplatin and fluorouracil as firstline therapy for advanced gastric cancer: a report of the V325 Study Group. J Clin Oncol. 2006;24:4991-7.

3. Cunningham D, Starling N, Rao S, Iveson T, Nicolson M, Coxon F, et al. Capecitabine and oxaliplatin for advanced esophagogastric cancer. N Engl J Med. 2008;358:36-46.

4. Shirasaka T, Nakano K, Takechi T, Satake H, Uchida J, Fujioka A, et al. Antitumor activity of $1 \mathrm{M}$ tegaful-0.4M 5-chloro-2,4dihydroxypyridine-1M potassium oxonate (S-1) against human colon carcinoma orthotopically implanted into nude rats. Cancer Res. 1996;56:2602-6.

5. Sakata Y, Ohtsu A, Horikoshi N, Sugimachi K, Mitachi Y, Taguchi T. Late phase II study of novel oral fluoropyrimidine anticancer drug S-1 (1 M tegafur- $0.4 \mathrm{M}$ gimestat-1M otastat potassium) in advanced gastric cancer patients. Eur $\mathrm{J}$ Cancer. 1998;34:1715-20.

6. Koizumi W, Tanabe S, Saigenji K, Ohtsu A, Boku N, Nagashima F, et al. Phase I/II study of S-1 combined with cisplatin in patients with advanced gastric cancer. Br J Cancer. 2003;89:2207-12.

7. Boku N, Yamamoto S, Fukuda H, Shirao K, Doi T, Sawaki A, et al. Fluorouracil versus combination of irinotecan plus cisplatin versus S-1 in metastatic gastric cancer: a randomized phase 3 study. Lancet Oncol. 2009;10:1063-9.

8. Koizumi W, Narahara H, Hara T, Takagane A, Akiya T, Takagi $\mathrm{M}$, et al. S-1 plus cisplatin versus S-1 alone for first-line treatment of advanced gastric cancer (SPIRITS trial): a phase III trial. Lancet Oncol. 2008;9:215-21. 
9. Ishigami S, Natsugoe S, Nakajo A, Matsumoto M, Uenosono Y, Arigami T, et al. Salvage gastrectomy following a combination of biweekly paclitaxel and S-1 for stage IV gastric cancer. J Gastrointest Surg. 2008;12:1370-5.

10. Fujitani K, Narahara H, Takiuchi H, Tsujinaka T, Satomi E, Gotoh M, et al. Phase I and pharmacokinetic study of S-1 combined with weekly paclitaxel in patients with advanced gastric cancer. Oncology. 2005;69:414-20.

11. Takiuchi H, Narahara H, Tsujinaka T, Gotoh M, Kawabe S, Katsu K, et al. Phase I study of S-1 combined with irinotecan (CPT-11) in patients with advanced gastric cancer (OGSG 0002). Jpn J Clin Oncol. 2005;35:520-5.

12. Therasse P, Arbuck SG, Eisenhauer EA, Wanders J, Kaplan RS, Rubinstein L, et al. New guidelines to evaluate the response to treatment in solid tumors. European Organization for Research and Treatment of Cancer, National Cancer Institute of the United States, National Cancer Institute of Canada. J Natl Cancer Inst. 2000;92:205-16.

13. Japanese Gastric Cancer Association. Japanese Classification of Gastric Carcinoma-2nd English Edition-response assessment of chemotherapy and radiotherapy for gastric carcinoma: clinical criteria. Gastric Cancer. 2001;4:1-8.

14. Greene FL, Page DL, Fleming ID, Fritz AG, Balch CM, Haller DG, et al. American Joint Committee on Cancer Staging Manual. 6th ed. New York: Springer; 2002. p. 99-103.

15. Japanese Gastric Cancer Association. Japanese classification of gastric carcinoma. 13th ed. Tokyo: Kanehara \& co., Ltd.; 1998 (in Japanese).

16. Ohashi M, Kanda T, Kobayashi T, Hirota M, Hayami M, Yajima $\mathrm{K}$, et al. Phase II study of weekly paclitaxel following fixed three cycles of S-1-based chemotherapy for advanced gastric cancer. Hepatogastroenterology. 2011;58:652-8.
17. Iwahashi M, Nakamori M, Tani M, Yamaue H, Sakaguchi S, Nakamura $\mathbf{M}$, et al. Complete response of highly advanced gastric cancer with peritoneal dissemination after new combined chemotherapy of S-1 and low-dose cisplatin: report of a case. Oncology. 2001;61:16-22.

18. Yoshimizu N, Saikawa Y, Kubota T, Yoshimizu N, Saikawa Y, Kubota $\mathrm{T}$, et al. Complete response of a highly advanced gastric carcinoma to preoperative chemoradiotherapy with S-1 and lowdose cisplatin. Gastric Cancer. 2003;6:185-90.

19. Yoshida I, Sakurai Y, Komori Y, Tonomura S, Masui T, Shoji M, et al. Successful downstaging by S-1-based chemotherapy followed by surgical resections for gastric carcinoma with extensive distant lymph node metastasis-report of two cases and a review of cases with surgical resection after downstaging by S-1-based chemotherapy. Hepatogastroenterology. 2005;52:978-84.

20. Sano T, Sasako M, Yamamoto S, Nashimoto A, Kurita A, Hiratsuka $\mathrm{M}$, et al. Gastric cancer surgery: morbidity and mortality results from a prospective randomized controlled trial comparing D2 and extended para-aortic lymphadenectomy: Japan Clinical Oncology Group Study 9501. J Clin Oncol. 2004;22:2767-73.

21. Degiuli M, Sasako M, Ponti A, Italian Gastric Cancer Study Group. Morbidity and mortality in the Italian Gastric Cancer Study Group randomized clinical trial of D1 versus D2 resection for gastric cancer. Br J Surg. 2010;97:643-9.

22. Cuschieri A, Fayers P, Fielding J, Craven J, Bancewicz J, Joypaul $\mathrm{V}$, et al. Postoperative morbidity and mortality after D1 and D2 resections for gastric cancer: preliminary results of the MRC randomised controlled surgical trial. The Surgical Cooperative Group. Lancet. 1996;347:995-9.

23. Nashimoto A, Yabusaki H, Nakagawa S, Takii Y, Tsuchiya Y, Otsuo T. Preoperative chemotherapy S-1 and cisplatin for highly advanced gastric cancer. Anticancer Res. 2009;29:4689-96. 